\title{
Macrocolonies (Granules) Formation as a Cause of False-Negative Results in the MGIT 960 System: Cause Analysis and Correlation with Mycobacterial Species
}

\author{
Xia Yu, Liping Zhao, Guanglu Jiang, Yifeng Ma, Weimin Li, and Hairong Huang \\ National Clinical Laboratory on Tuberculosis, Beijing Key laboratory on Drug-Resistant Tuberculosis Research, \\ Beijing Chest Hospital, Capital Medical University, Beijing Tuberculosis and Thoracic Tumor Institute, Beijing 101149, China
}

Correspondence should be addressed to Hairong Huang; hairong.huangcn@gmail.com

Received 25 May 2015; Revised 6 September 2015; Accepted 28 September 2015

Academic Editor: Urszula Demkow

Copyright (C) 2015 Xia Yu et al. This is an open access article distributed under the Creative Commons Attribution License, which permits unrestricted use, distribution, and reproduction in any medium, provided the original work is properly cited.

Background. The viable mycobacterial bacilli can sometimes form granules in the Mycobacterium Growth Indicator Tube (MGIT) to produce instrument-negative outcomes when BACTEC MGIT 960 culture is performed. The cause of this phenomenon has never been analyzed. Methods. Thirty-one instrument-negative, granule presenting MGIT vials were collected for conducting acid-fast staining and also liquid and solid subculture. Species identification and drug susceptibility test were performed with the recovered strains. Cultivation test was done by inoculating small amount of bacilli into the MGIT vials. Results. Twenty-four and twentynine of the tested MGIT vials were smear and culture positive, respectively. In total, 18, 4, and 7 of the cultivated strains were identified as Mycobacterium tuberculosis complex, M. intracellulare, and M. xenopi, respectively. When a limited amount of bacilli was inoculated, the granule formation was observed for M. xenopi strains in the MGIT system. Conclusions. The granules observed in the instrument-negative MGIT vials consisted of viable bacilli, which emphasized the need of visual inspection to increase recovery rate. Limited bacterial load and specific species might be the cause of granule forming.

\section{Introduction}

Bacterial culturing is substantially more sensitive than smear microscopy to diagnose tuberculosis (TB) [1], while liquid culture is faster and more sensitive than solid culture [25]. The BACTEC MGIT 960 system (Becton, Dickinson and Company, Franklin Lakes, NJ, USA) is a widely used commercial automatic culture system based on the bacterial culturing in broth. A situation may occasionally arise when using MGIT 960 system, that is, the presence of visible granules (characterized by a scanty number of round yellowpigmented appearance and approximately $1 \mathrm{~mm}$ size), in some instrument-negative vials. Even after shaking the MGIT tubes, these granules maintain their shapes rather than forming a homogenously turbid solution. To better understand this outcome, we collected such kind of vials to analyze the features and cause of these granules.

\section{Material and Methods}

2.1. Sample Collection. Mycobacterial culturing by MGIT 960 system was performed for the 2,410 clinical specimens, collected during January 2010 to December 2010 at the National Tuberculosis Clinical Laboratory of Beijing Chest Hospital. From these samples, 1,338 were flagged as culture positive by the instrument.

2.2. Smear Microscopy Examination. Granules in the MGIT vials were harvested by centrifugation and swabbed directly with sterile Pasteur pipettes onto slides to prepare smears. The slides were then stained using the Ziehl-Neelsen method, according to the protocol by World Health Organization (WHO) [6].

2.3. Subculture. For each of the tested MGIT vials, the tube was briefly centrifuged and the sediment was resuspended in 


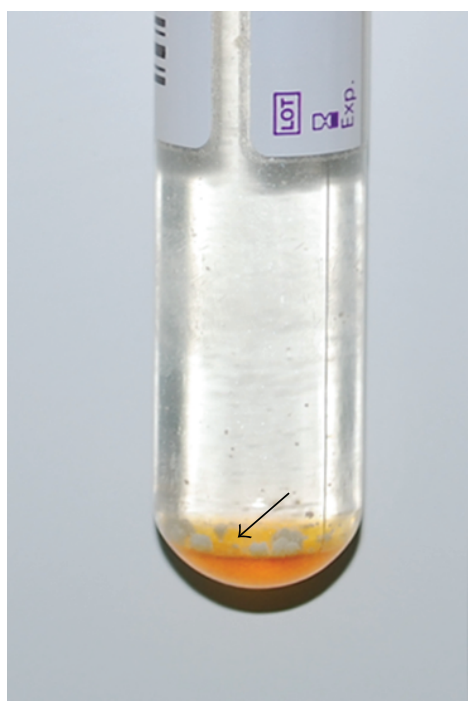

(a)

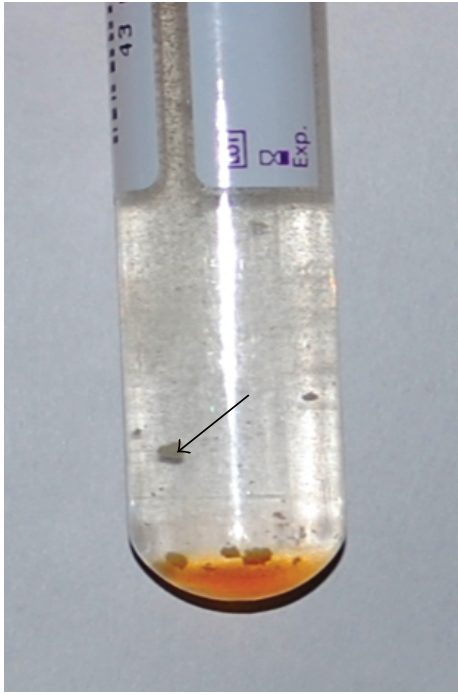

(b)

FIGURE 1: Granules in the instrument-negative MGIT vials. (a) Granules before shaking. (b) Granules after shaking.

$1 \mathrm{~mL}$ of $7 \mathrm{H} 9$ broth. Half of the suspension was then inoculated into a fresh MGIT vial while another half was inoculated onto a fresh LJ medium slant for mycobacterial culture.

2.4. Species Identification. The $16 \mathrm{~S}-23 \mathrm{~S}$ rRNA internal transcribed spacer (ITS) regions of the strains were sequenced after amplification by PCR to categorize the strains into species, as mentioned elsewhere [7-9].

2.5. Drug Susceptibility Test (DST). The DST was performed by the MGIT 960 system as recommended [10, 11]. The tested drugs included isoniazid (INH), rifampin (RIF), streptomycin (SM), and ethambutol (EMB).

2.6. Seeded Culture Studies. Mycobacterium tuberculosis strain H37Rv (ATCC strain 27294) and 8 aforementioned isolates acquired from the granules' subculture were chosen by the method of stratified cluster sampling [12]. The 8 strains included $2 M$. tuberculosis complex (MTC) strains, $3 M$. xenopi strains, and 3 M. intracellulare strains. The isolates were grown on LJ slants at $37^{\circ} \mathrm{C}$ for three weeks; then, the bacterial suspensions were prepared using $0.5 \%$ (vol/vol) Tween 80 in $0.9 \%$ sodium chloride with turbidity equal to the McFarland number 1 turbidity standard. Next, the suspensions were diluted to $10^{-5}, 10^{-6}, 10^{-7}$, and $10^{-8}$ of the McFarland number 1 turbidity standards. Aliquots (approximately $100 \mu \mathrm{L}$ ) of each preparation were inoculated into new MGIT vials in duplicate. The MGIT 960 culture was considered positive if the instrument signaled positive and the following microscopic examination proved AFB existence. If the instrument did not signal positive until after 6 weeks of incubation, the tubes were visually inspected for the presence of granules. All the inoculated MGIT vials were centrifuged after the culture completion and sediments were used for acid-fast staining. The existence of viable mycobacteria was proven for all the instrument-negative vials (including the granule forming vials) by inoculating the sediments into new MGIT960 vials for another culture.

2.7. Statistics. Chi-square test was performed for comparing the species composition between instrument positive vials and granule forming vials. A value of $P<0.05$ was considered statistically significant. The statistical analysis was carried out using SPSS version 13.0.

2.8. Ethical Considerations. The study was approved by the ethical committee of Beijing Chest Hospital. The data was accessible only to study personnel.

\section{Results}

3.1. Microscopy Examination and Culture. A total of 31 instrument-negative MGIT vials, with visible granules, were collected (Figure 1). Among these, the GI values for 27 vials were zero and were 40,5, 63, and 55 for the other 4 vials. All the granules vials remained instrument-negative even after elongating the incubation time for 2 more weeks in the MGIT960 system. The following microscopic examination, MGIT 960 subculture and LJ medium subculture produced positive outcomes for 24,25 , and 29 vials, respectively.

3.2. Species Identification Outcomes. Among the 1338 instrument flagged-positive MGIT vials, the species composition was as follows: $\operatorname{MTC}(n=1267), M$. intracellulare $(n=31)$, M. abscessus $(n=17)$, M. kansasii $(n=11)$, M. gordonae $(n=6)$, M. avium $(n=3)$, M. xenopi $(n=1), M$. malmoense $(n=1)$, and M. scrofulaceum $(n=1)$. Among the strains recovered from granules, MTC, $M$. intracellulare, and 
TABLE 1: The drug susceptibility test outcomes of 18 MTC isolates with granules forming to four first-line antituberculosis drugs.

\begin{tabular}{lcccc}
\hline \multicolumn{4}{c}{ Antituberculosis drugs } & \multicolumn{2}{c}{ Number of isolates } \\
SM & INH & RIF & EMB & 7 \\
\hline S & S & S & S & 5 \\
R & R & R & S & 2 \\
R & S & S & S & 2 \\
R & R & R & R & 1 \\
R & R & S & S & 1 \\
S & R & S & S & \\
\hline
\end{tabular}

S: susceptible; R: resistant.

M. xenopi accounted for $62.07 \%\left(18 / 29, \chi^{2}=53.58, P<\right.$ $0.001), 13.79 \%\left(4 / 29, \chi^{2}=14.99, P<0.001\right)$, and $24.14 \%$ (7/29, $\left.\chi^{2}=282.50, P<0.001\right)$ of the strains, respectively.

3.3. Drug Susceptibility Test Outcomes. Among the 18 aforementioned MTC strains, 7 strains were sensitive to all the four tested drugs (INH, RIF, SM, and EMB) and 7 strains were classified as MDR strains whereas the remaining 4 strains demonstrated resistance patterns other than MDR. The drug susceptibility test outcomes of 18 MTC isolates were listed in Table 1.

3.4. Inoculation Test. The outcomes of inoculation test for 3 M. xenopi, 3 M. intracellular, and 2 MTC strains as well as for M. tuberculosis H37Rv are listed in Table 2. The vials signaled as culture negative, with or without granule formation, were all culture positive after subculturing the sediments into the new MGIT vials.

\section{Discussion}

The BACTEC MGIT 960 instrument is a fully automated, noninvasive system that is suitable for the detection of tuberculosis and other mycobacterial species. We observed that some instrument-negative tubes presented visible granules after 6 weeks of incubation; however, extending the incubation time to 8 weeks did not produce any positive outcome. Bacteriological examination proved that the granules consisted of viable bacilli. Meanwhile, other studies have also reported failure in growth detection by MGIT 960 [1315]. Although the manufacturer suggested either subculturing the vials with granules or conducting acid-fast stains and treating the specimen as presumptive positives (provided that either test is positive) [16]. The reason for granule formation is not well understood so far: dead bacilli cannot be the cause because 29 of the 31 subcultured vials with granules achieved positive culture outcomes. Therefore, according to our work, visual inspection of the negative vials could further increase approximately $1 \%$ of bacilli recovery rate for MGIT 960 culture.

Our DST outcomes suggested that the granule formation may not be related with drug resistance patterns, since the MDR-TB proportion is similar to that of the overall isolates in the same laboratory (approximately $1 / 3$ of the test strains are MDR strains). Species identification demonstrated that 11 strains $(11 / 29,37.93 \%)$ were NTM strains, which suggested that the NTM is not the exclusive explanation for granule formation. However, our data did show that some types of NTM might have a better opportunity to form granules than others, because $M$. intracellulare or $M$. xenopi accounted for much higher percentages of granule forming isolates than the general culture positive isolates $(P<0.001)$. On the other hand, MTC isolates demonstrated opposite tendency for granule formation $(P<0.001)$. A similar study demonstrated that $94 \%$ (129/137) of instrumentnegative outcome was caused by NTM, and the most frequent NTM was $M$. avium complex and $M$. gordonae in the United States [14]. However, due to limited total number of tested strains in our study, the possibility that this result was due to sampling error cannot be confidently excluded.

In the inoculation assay, only the $3 \mathrm{M}$. xenopi strains produced granules when inoculated with limited bacterial loads. Such outcomes not only proved that granule formation might be a specific growing characteristic for $M$. xenopi but also demonstrated that low bacterial load might be a synergetic factor. Piersimoni et al., also found that due to granular pattern of growth (which keeps oxygen consumption below the detection threshold), MGIT 960 can fail in the detection of M. xenopi bacilli [13]. The MTC strains and M. intracellulare strains failed to produce granules with low inoculation bacterial loads, demonstrating that some other factors may also exist. Among the related patients with granule forming outcomes, except for one with unclear medical record, all other patients were receiving anti-TB treatment when the samples were collected. The treatment duration lasted from 1 month to more than 2 years. Therefore, it is likely that the administration of anti-TB therapy (probably together with a low bacterial load) can be a key factor for the development of granules. Among the granule forming vials, 3 with MTC granules and 2 with $M$. xenopi granules originated from same patients within a very short time period. We guess the bacterial loads in specimens might have been similar during the short period.

Our experiment also demonstrated that acid-fast staining increased the chances to identify the AFB in the granules, as $24(24 / 29,82.76 \%)$ of the granule forming vials were smear positive. Since it is possible to lose AFB staining while preparing smear slides with liquid broth, improving the smear preparation process can also increase the AFB detection sensitivity.

\section{Conclusions}

The granules observed in the instrument-negative MGIT vials consist of viable bacilli; therefore, visual inspection for granules is suggested for better yield. Limited bacterial load and specific species can also cause granule formation during MGIT 960 culturing. 
TABLE 2: The MGIT culture outcomes when inoculated with different bacterial loads in duplicate (by tubes).

\begin{tabular}{|c|c|c|c|c|c|}
\hline Number and species & $\begin{array}{l}\text { Diluted McFarland } \\
\text { turbidity standard }\end{array}$ & $\begin{array}{l}\text { Culture } \\
\text { positive }\end{array}$ & $\begin{array}{l}\text { Turn-around time (days) } \\
\quad(\text { means } \pm \text { SD) }\end{array}$ & $\begin{array}{c}\text { Culture negative with } \\
\text { granules forming }\end{array}$ & $\begin{array}{c}\text { Culture negative without } \\
\text { granules forming }\end{array}$ \\
\hline \multirow{4}{*}{3 M. xenopi strains } & $10^{-5}$ & $6 / 6$ & $9.67 \pm 1.03$ & $0 / 6$ & $0 / 6$ \\
\hline & $10^{-6}$ & $2 / 6$ & $13.5 \pm 0.71$ & $4 / 6$ & $0 / 6$ \\
\hline & $10^{-7}$ & $1 / 6$ & $26.3 \pm 0.57$ & $5 / 6$ & $0 / 6$ \\
\hline & $10^{-8}$ & $0 / 6$ & - & $6 / 6$ & $0 / 6$ \\
\hline \multirow{4}{*}{3 M. intracellulare strains } & $10^{-5}$ & $6 / 6$ & $9.17 \pm 1.47$ & $0 / 6$ & $0 / 6$ \\
\hline & $10^{-6}$ & $4 / 6$ & $11.75 \pm 1.26$ & $0 / 6$ & $2 / 6$ \\
\hline & $10^{-7}$ & $0 / 6$ & - & $0 / 6$ & $6 / 6$ \\
\hline & $10^{-8}$ & $0 / 6$ & - & $0 / 6$ & $6 / 6$ \\
\hline \multirow{4}{*}{$2 M T C$ strains } & $10^{-5}$ & $4 / 4$ & $11.00 \pm 0.82$ & $0 / 4$ & $0 / 4$ \\
\hline & $10^{-6}$ & $4 / 4$ & $15.00 \pm 0.81$ & $0 / 4$ & $0 / 4$ \\
\hline & $10^{-7}$ & $3 / 4$ & $25.00 \pm 1.00$ & $0 / 4$ & $1 / 4$ \\
\hline & $10^{-8}$ & $1 / 4$ & 36.00 & $0 / 4$ & $3 / 4$ \\
\hline \multirow{4}{*}{$\mathrm{H} 37 \mathrm{Rv}$} & $10^{-5}$ & $2 / 2$ & $10.00 \pm 0.00$ & $0 / 2$ & $0 / 2$ \\
\hline & $10^{-6}$ & $2 / 2$ & $13.50 \pm 0.71$ & $0 / 2$ & $0 / 2$ \\
\hline & $10^{-7}$ & $2 / 2$ & $25.00 \pm 1.41$ & $0 / 2$ & $0 / 2$ \\
\hline & $10^{-8}$ & $0 / 2$ & - & $0 / 2$ & $2 / 2$ \\
\hline
\end{tabular}

SD: standard deviations.

\section{Conflict of Interests}

The authors declare no conflict of interests.

\section{Author's Contribution}

Xia Yu and Liping Zhao carried out the experimental data and analysis and drafted the paper; Guanglu Jiang and Yifeng Ma carried out the drug susceptibility test; Weimin Li and Hairong Huang were responsible for data analysis; Hairong Huang was responsible for the conception of the study design and preparation of the paper. All authors read and approved the final paper.

\section{Acknowledgments}

The work was supported by the research funding from Infectious Diseases Special Project, Ministry of Health of China (2012ZX10003002), and Beijing Municipal Administration of Hospitals Clinical Medicine Development of Special Funding Support (ZYLX201304). The strains used in this project were obtained from the "Beijing Bio-Bank of Clinical Resources on Tuberculosis (D09050704640000)," Beijing Chest Hospital.

\section{References}

[1] L. M. Parsons, Á. Somoskövi, C. Gutierrez et al., "Laboratory diagnosis of tuberculosis in resource-poor Countries: challenges and opportunities," Clinical Microbiology Reviews, vol. 24, no. 2, pp. 314-350, 2011.

[2] W. K. Chew, R. M. Lasaitis, F. A. Schio, and G. L. Gilbert, "Clinical evaluation of the mycobacteria growth indicator tube (MGIT) compared with radiometric (Bactec) and solid media for isolation of Mycobacterium species," Journal of Medical Microbiology, vol. 47, no. 9, pp. 821-827, 1998.

[3] N. Hines, J. B. Payeur, and L. J. Hoffman, "Comparison of the recovery of Mycobacterium bovis isolates using the BACTEC MGIT 960 system, BACTEC 460 system, and Middlebrook 7H10 and 7H11 solid media," Journal of Veterinary Diagnostic Investigation, vol. 18, no. 3, pp. 243-250, 2006.

[4] D. S. G. Chan, Y. C. May, S. Wang, and L.-H. Sng, "An evaluation of the recovery of mycobacteria from urine specimens using the automated Mycobacteria Growth Indicator Tube system (BACTEC MGIT 960)," Journal of Medical Microbiology, vol. 57, part 1, no. 10, pp. 1220-1222, 2008.

[5] E. Tortoli, P. Cichero, C. Piersimoni, M. T. Simonetti, G. Gesu, and D. Nista, "Use of BACTEC MGIT 960 for recovery of mycobacteria from clinical specimens: multicenter study," Journal of Clinical Microbiology, vol. 37, no. 11, pp. 3578-3582, 1999.

[6] World Health Organization, Laboratory Service in Tuberculosis Control: Part II. Microscopy, World Health Organization, 1998.

[7] Y. Abed, C. Bollet, and P. de Micco, "Identification and strain differentiation of Mycobacterium species on the basis of DNA 16S-23S spacer region polymorphism," Research in Microbiology, vol. 146, no. 5, pp. 405-413, 1995.

[8] A. Roth, M. Fischer, M. E. Hamid, S. Michalke, W. Ludwig, and H. Mauch, "Differentiation of phylogenetically related slowly growing mycobacteria based on 16S-23S rRNA gene internal transcribed spacer sequences," Journal of Clinical Microbiology, vol. 36, no. 1, pp. 139-147, 1998.

[9] A. Sansila, P. Hongmanee, C. Chuchottaworn, S. Rienthong, D. Rienthong, and P. Palittapongarnpim, "Differentiation between Mycobacterium tuberculosis and Mycobacterium avium by amplification of the 16S-23S ribosomal DNA spacer," Journal of Clinical Microbiology, vol. 36, no. 9, pp. 2399-2403, 1998.

[10] F. Kontos, M. Maniati, C. Costopoulos et al., "Evaluation of the fully automated Bactec MGIT 960 system for the susceptibility 
testing of Mycobacterium tuberculosis to first-line drugs: a multicenter study," Journal of Microbiological Methods, vol. 56, no. 2, pp. 291-294, 2004.

[11] A. Krüüner, M. D. Yates, and F. A. Drobniewski, "Evaluation of MGIT 960-based antimicrobial testing and determination of critical concentrations of first- and second-line antimicrobial drugs with drug-resistant clinical strains of Mycobacterium tuberculosis," Journal of Clinical Microbiology, vol. 44, no. 3, pp. 811-818, 2006.

[12] C. Piersimoni, D. Nista, S. Bornigia, and G. Gherardi, "Unreliable detection of Mycobacterium xenopi by the nonradiometric Bactec MGIT 960 culture system," Journal of Clinical Microbiology, vol. 47, no. 3, pp. 804-806, 2009.

[13] J. A. Peña, M. J. Ferraro, C. G. Hoffman, and J. A. Branda, "Growth detection failures by the nonradiometric Bactec MGIT 960 mycobacterial culture system," Journal of Clinical Microbiology, vol. 50, no. 6, pp. 2092-2095, 2012.

[14] P. Sharma, K. Sharma, D. Singh, S. Verma, S. Mahajan, and A. Kanga, "Failure of the MGIT 960 culture system in the detection of Mycobacterium tuberculosis," International Journal of Tuberculosis and Lung Disease, vol. 18, no. 12, p. 1525, 2014.

[15] Becton Dickinson and Company, BBL MGIT Mycobacterial Growth Indicator Tube Package Insert (7-ml Tube), Becton, Dickinson and Company, Sparks, Md, USA, 2011.

[16] National Technic Steering Group of the Epidemiological Sampling Survey for Tuberculosis, "Report on fourth national epidemiological sampling survey for tuberculosis," Chinese Journal of Tuberculosis and Respiratory Diseases, vol. 25, no. 1, pp. 3-7, 2002. 


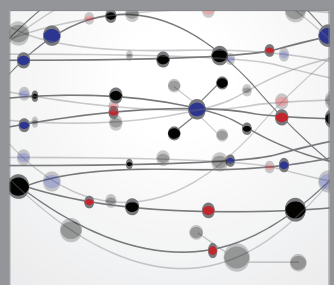

The Scientific World Journal
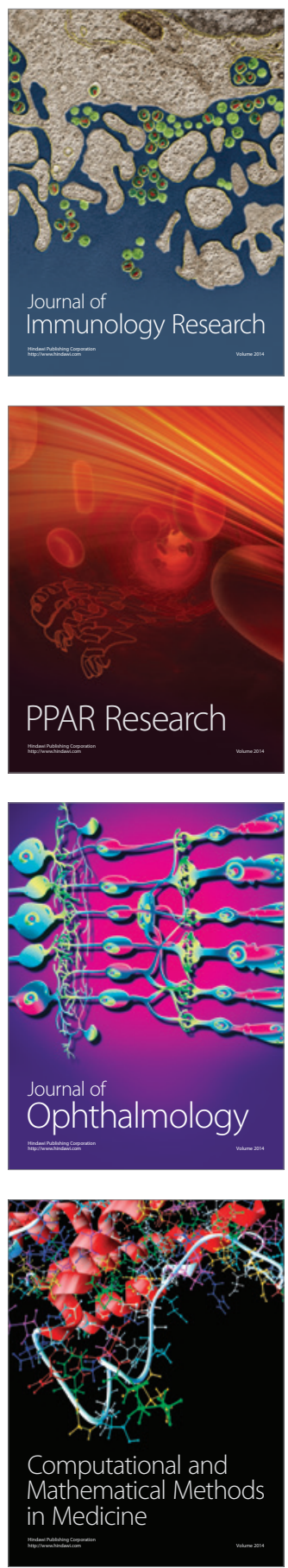

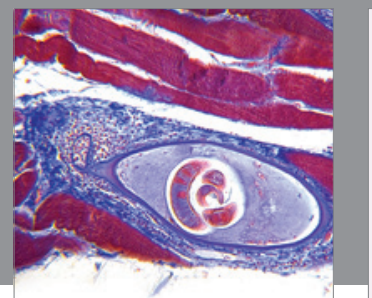

Gastroenterology

Research and Practice
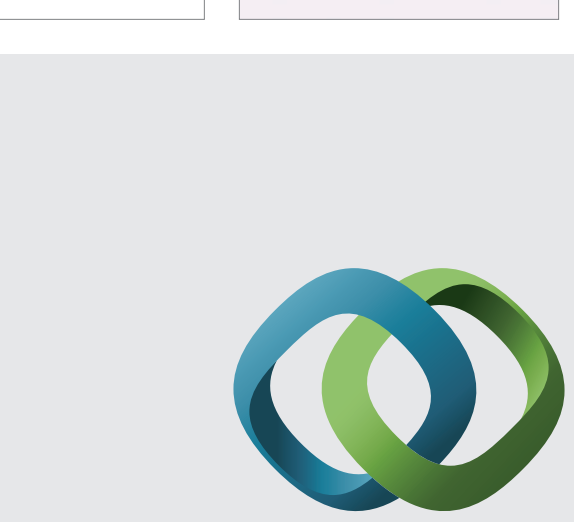

\section{Hindawi}

Submit your manuscripts at

http://www.hindawi.com
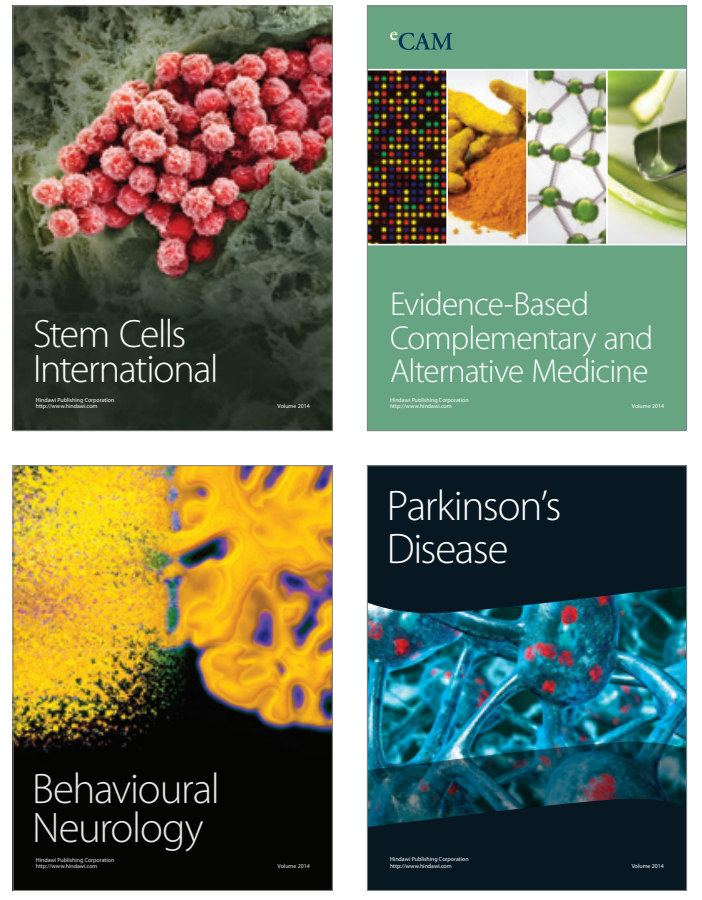
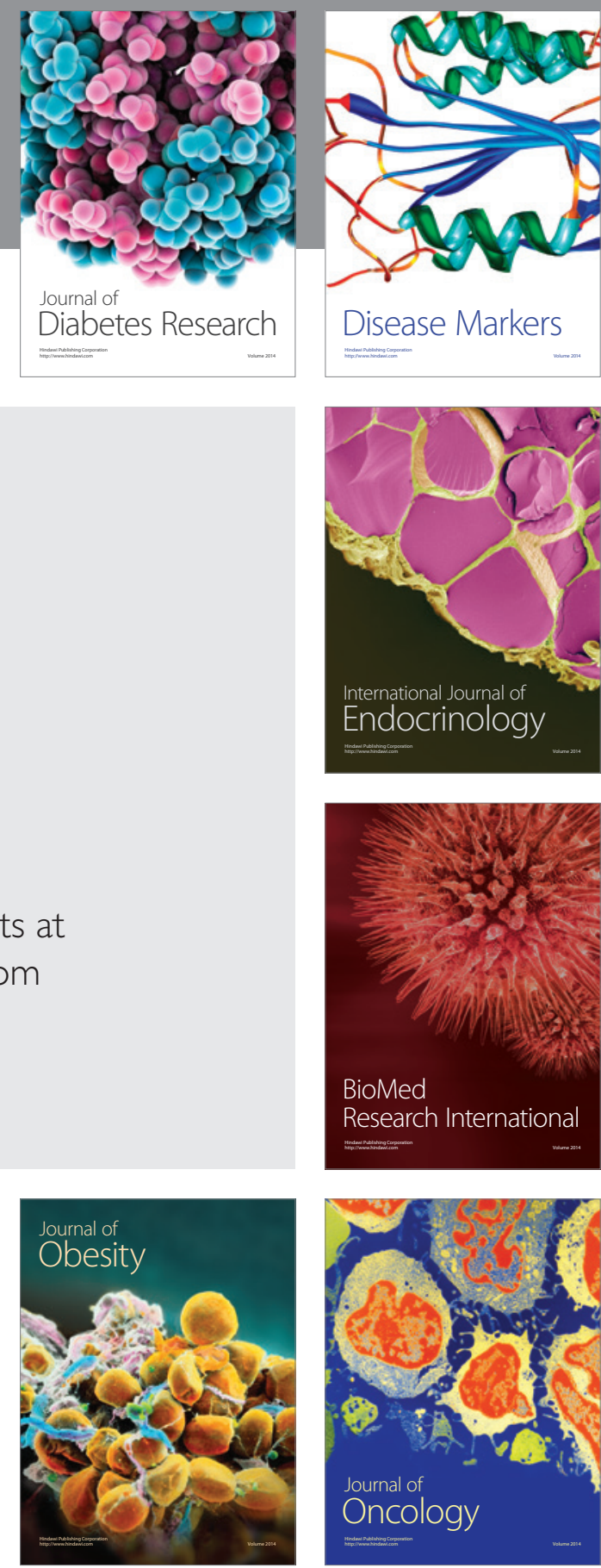

Disease Markers
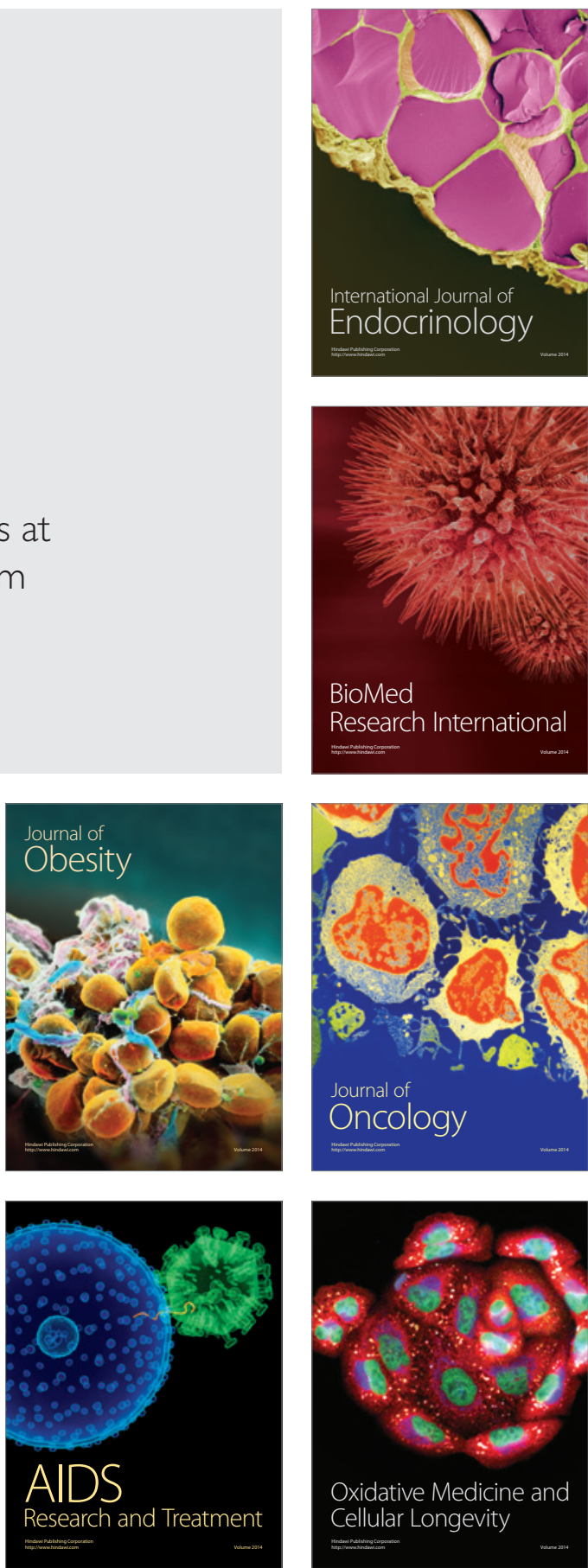